\title{
LETTER
}

\section{Extravascular lung water and pulmonary vascular permeability index may inadvertently delay the identification of acute respiratory distress syndrome}

\author{
Zong-Yu Wang and Yu Bai* \\ See related research by Kushimoto et al., http://ccforum.com/content/16/6/R232
}

In a recent issue of Critical Care, Kushimoto and colleagues [1] reported a multicenter cohort study that showed pulmonary vascular permeability index (PVPI) to be a practical diagnostic parameter to recognize acute lung injury/acute respiratory distress syndrome (ALI/ ARDS) in pulmonary edema. Their study deserves to be appreciated. The transpulmonary thermodilution technique allows an accurate bedside estimation of increased pulmonary vascular permeability, which is the physiopathologic hallmark of ALI/ARDS [2], facilitating the validity of diagnosis. However, the specific quantitative diagnostic parameter perhaps inadvertently compromises the effort to identify ARDS early.

Firstly, the relatively limited availability of PiCCO probably restricts the comprehensive application of PVPI. Secondly, some patients with mild ARDS may manifest only mild hypoxemia, which requires only non-invasive mechanical ventilation. In this case, it seems cumbersome to obtain extravascular lung water index (EVLWI) and PVPI for diagnosis. To do that, in fact, hinders rapid recognition of mild ARDS to some extent. Thirdly, the cutoff of EVLWI $(\geq 10 \mathrm{~mL} / \mathrm{kg})$ exhibited low sensitivity (63\%) [3], as did the PVPI cutoff in this study. Thus, EVLWI and PVPI are combined into a diagnostic composite to achieve optimum sensitivity and specificity by altering the threshold of each parameter. Nevertheless, we are more hopeful of establishing the stratification criteria, which keep the composite of EVLWI and PVPI as a standard for confirming a diagnosis of ARDS, while American-European Consensus Conference criteria or the Berlin definition is treated as a selection criterion to quickly screen for patients with hypoxemia in clinical practice.

\section{Authors' response}

Shigeki Kushimoto, Satoshi Yamanouchi, Tomoyuki Endo and Takashi Tagami

We would like to thank Wang and Bai for their discerning comments on our study [1]. We agree with their view that PVPI combined with EVLWI is considered to be a quantitative diagnostic parameter to facilitate a valid and definitive diagnosis of ARDS.

In regard to their first concern, the devices required for the evaluation of EVLWI and PVPI by the thermodilution method are commercially available but are specialized devices, and the procedures are not non-invasive. The values provide important quantitative data that facilitate

*Correspondence: baiyu0617@163.com

Department of Intensive Care Unit, Peking University Third Hospital,

49 North Garden Road, Haidian District, Beijing, China accurate bedside estimation of increased extravascular water secondary to increased permeability, which is the physiopathologic hallmark of ARDS; however, this technique may prevent early detection of mild ARDS if the diagnosis is based on the values and the measurements are mandatory. Measurement of EVLWI and PVPI at the bedside is practical; however, this measurement is not included in the Berlin definition, because it is not available at all institutions and hence cannot be made mandatory [4].

In regard to their concern about the sensitivity of the EVLWI cutoff values ( $>10 \mathrm{~mL} / \mathrm{kg}$ ) for ARDS, we recently demonstrated that, in order to distinguish between normal lungs and lungs with ARDS, the value is approximately $10 \mathrm{~mL} / \mathrm{kg}$, as suggested by researchers in two previous studies [5,6]. At present, the EVLWI cutoff value 
of $10 \mathrm{~mL} / \mathrm{kg}$ may be appropriate, and further studies are required to determine a value with sufficient sensitivity and specificity.

We believe that direct measurement of PVPI and EVLWI can help determine the accurate clinical condition of ARDS and can be incorporated into the future definition of ARDS.

\section{Abbreviations}

ALI, acute lung injury; ARDS, acute respiratory distress syndrome; EVLWI, extravascular lung water index; PVPI, pulmonary vascular permeability index.

\section{Competing interests}

The authors declare that they have no competing interests.

\section{Authors' contributions}

Z-YW and YB developed the concept, drafted the manuscript, and have read and approved the final manuscript.

Published: 20 March 2013

\section{References}

1. Kushimoto S, Taira Y, Kitazawa Y, Okuchi K, Sakamoto T, Ishikura H, Endo T, Yamanouchi S, Tagami T, Yamaguchi J, Yoshikawa K, Sugita M, Kase Y, Kanemura T, Takahashi H, Kuroki Y, Izumino H, Rinka H, Seo R, Takatori M, Kaneko T, Nakamura T, Irahara T, Saito N, Watanabe A, PiCCO Pulmonary
Edema study group: The clinical usefulness of extravascular lung water and pulmonary vascular permeability index to diagnose and characterize pulmonary edema: a prospective multicenter study on the quantitative differential diagnostic definition for acute lung injury/acute respiratory distress syndrome. Crit Care 2012, 16:R232.

2. Sakka SG: Extravascular lung water in ARDS patients. Minerva Anestesiol 2012, Dec 20. [Epub ahead of print].

3. LeTourneau JL, Pinney J, Phillips CR: Extravascular lung water predicts progression to acute lung injury in patients with increased risk* . Crit Care Med 2012, 40:847-854.

4. ARDS Definition Task Force, Ranieri VM, Rubenfeld GD, Thompson BT, Ferguson ND, Caldwell E, Fan E, Camporota L, Slutsky AS: Acute respiratory distress syndrome: the Berlin Definition. JAMA 2012, 307:2526-2533.

5. Tagami T, Kushimoto S, Yamamoto Y, Atsumi T, Tosa R, Matsuda K, Oyama R, Kawaguchi T, Masuno T, Hirama H, Yokota H: Validation of extravascular lung water measurement by single transpulmonary thermodilution: human autopsy study. Crit Care 2010, 14:R162.

6. Tagami T, Sawabe M, Kushimoto S, Marik PE, Mieno MN, Kawaguchi T, Kusakabe T, Tosa R, Yokota H, Fukuda Y: Quantitative diagnosis of diffuse alveolar damage using extravascular lung water. Crit Care Med 2013, 79:274-284.

doi:10.1186/cc12542

Cite this article as: Wang ZY, Bai $Y$ : Extravascular lung water and pulmonary vascular permeability index may inadvertently delay the identification of acute respiratory distress syndrome. Critical Care 2013, 17:420. 